\title{
Mangiferin Mitigates Lipopolysaccharide-Induced Lung Injury by Inhibiting NLRP3 Inflammasome Activation
}

\section{Ning Li* \\ Rui Xiong* \\ Ruyuan $\mathrm{He}$ \\ Bohao Liu \\ Bo Wang \\ Qing Geng}

Department of Thoracic Surgery, Renmin Hospital of Wuhan University, Wuhan, 430060, People's Republic of China

*These authors contributed equally to this work
Correspondence: Bo Wang; Qing Geng Department of Thoracic Surgery, Renmin Hospital of Wuhan University, Jiefang Road 238, Wuhan, 430060, People's Republic of China

Email rmh_wb@whu.edu.cn;

gengqingwhu@whu.edu.cn
Scope: Mangiferin (MF) is a natural phytopolyphenol, which displays potential pharmacological properties involving antibacterial, anti-inflammation, antioxidant and anti-tumor. However, little is known about the roles of MF in lung injury. The aim of this study is to demonstrate the modulatory effects and molecular mechanisms by which MF operates in sepsis-induced lung injury.

Methods and Results: To examine the protective properties of MF, an in vivo model of lipopolysaccharide (LPS)-induced lung injury in mice and an in vitro model of LPS-treated J774A.1 cells were established, respectively. The results revealed that MF treatment significantly relieved LPS-induced pathological injury and inflammatory response in murine lung tissues. Meanwhile, MF treatment also inhibited nucleotide-binding oligomerization domain (NOD)-like receptor family, pyrin domain-containing protein 3 (NLRP3) inflammasome activation and pyroptosis induced by LPS. In macrophage-specific NLRP3 deficiency mice treated with LPS, MF showed little protective effects. NLRP3 overexpression by adenovirus could also offset the beneficial effects of MF in LPS-treated J774A.1 cells. Furthermore, we found that MF could suppress the expression of NLPR3 and pyroptosis of macrophages by inhibiting the nuclear translocation of the nuclear factor- $\kappa \mathrm{B}(\mathrm{NF}-\kappa \mathrm{B})$ subunits P50 and P65.

Conclusion: MF protects against lung injury and inflammatory response by inhibiting NLRP3 inflammasome activation in a NF-kB-dependent manner in macrophages, which provides a promising therapeutic candidate for the treatment of lung injury.

Keywords: mangiferin, lung injury, NLRP3, P65

\section{Introduction}

Acute lung injury (ALI) is usually caused by respiratory bacteria or virus infection, sepsis and shock, which is a life-threatening condition featured by pulmonary edema, uncontrolled inflammatory response and increased vascular permeability. ${ }^{1,2}$ During ALI, inflammatory response-activated neutrophils and macrophages can infiltrate into lung tissues and release cytokines, activating local pro-inflammatory reaction. ${ }^{3}$ Innate immune signaling receptor NOD-like receptor family pyrin domain containing 3 (NLRP3) could be triggered by various danger signals including damage-related molecular patterns (DAMPs) and pathogen associated molecular patterns (PAMPs). ${ }^{4}$ NLRP3 is the core assembly of inflammasome, the activation of which may give rise to caspase 1-mediated proteolytic activation of the interleukin-18 (IL-18) and IL-1 $\beta$, and induce pyroptosis. ${ }^{5}$ Our 
team recently reported that NLRP3 inflammasome activation by mitochondrial DNA is one of the critical mechanisms contributing to sepsis-induced ALI. ${ }^{6}$ And pharmacological inhibition or genetic ablation of NLRP3 activation could lead to potent beneficial effects in lung diseases, especially in sepsis-induced ALI. ${ }^{7}$ Hence, targeted inhibition of NLRP3 inflammasome activation or its upstream regulation molecules in lung tissues could be a possible strategy for the prevention of ALI.

Mangiferin (MF) is a xanthone present in mango fruit and other different parts including stalks, peel, barks, leaves, as well as kernel. ${ }^{8} \mathrm{MF}$ possesses multiple pharmacologic properties including antidiabetic, anti-inflammatory, antiviral, antioxidative, antiaging, immunomodulatory, cardioprotective and analgesic effects. ${ }^{9,10}$ In particular, MF also displays potent inhibitory effect on NLRP3 inflammasome in some certain diseases. For instance, MF could relieve blast-induced traumatic brain injury by inactivating NLRP3 inflammasome. ${ }^{11}$ In endothelial cells, MF is involved in the inhibition of endothelial dysfunction by blocking endoplasmic reticulum stress-associated NLRP3 inflammasome activation. ${ }^{12}$ However, previous studies paid little attention to the beneficial effects of MF on lung diseases and sepsis. Therefore, in the present study, we intended to explore whether MF pretreatment could prevent ALI, and meanwhile to uncover the potential mechanisms of MF in ALI.

\section{Experimental Section}

\section{Materials}

Mangiferin (MF), purity $>99.84 \%$, was purchased from the Shanghai Winherb Medical Co. (Shanghai, China). Lipopolysaccharide (LPS) (Escherichia coli 055:B5) was obtained from Sigma-Aldrich (St. Louis, MO, USA). Anti- $\beta$-actin (ab6276), anti-NLRP3 (ab263899), anti-ASC (ab151700) and anti-GSDMD (ab219800) were obtained from Abcam (Cambridge, MA, USA).

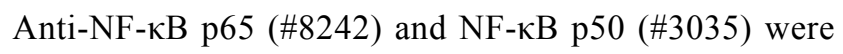
provided by Cell Signalling Technology (Danvers, MA, USA). Dulbecco's modified Eagle's medium (DMEM) and fetal bovine serum (FBS) were obtained from Invitrogen-Gibco (Grand Island, NY). The secondary antibody was provided by LI-COR Biosciences (Lincoln, United States). All chemicals used in this study were of analytical grade.

\section{Animals and Treatments}

All experiments were conducted in accordance with the NIH Guide for the Care and Use of Laboratory Animals, and were approved by Animal Care and Use Committee of Wuhan University. C57BL/6J male mice (8-10 weeks old, weight: $25.3 \pm 1.6 \mathrm{~g}$ ) were provided by the Institute of Laboratory Animal Science, Chinese Academy of Medical Sciences (Beijing, China). Macrophage-specific NLRP3 deficient mice with C57BL/6 background (8-10 weeks old, weight: $25.1 \pm 1.5 \mathrm{~g})$ were generated by crossing NLRP3-floxed mice (NLRP3 ${ }^{\mathrm{f} / \mathrm{f}}$ ) with Lyz2-Cre transgenic mice. All experimental animal in our study were fed in a specific-pathogen-free (SPF) environment (humidity 50 $\pm 3 \%$, temperature $22-25^{\circ} \mathrm{C}$ ). As described previously, ${ }^{6}$ to establish the murine ALI model, LPS was dissolved in 50 $\mu \mathrm{L}$ sterile saline and instilled intratracheally at a dose of $5 \mathrm{mg} / \mathrm{kg}$. An isovolumetric sterile saline was instilled intratracheally in mice from the control group. Twelve hours later, the left lungs were excised when mice were sacrificed under deep anesthesia by cervical dislocation. Formalin was instilled in the trachea to expand the alveoli. All operations and subsequent analyses were carried out in a blind fashion for all groups.

\section{Dosage Information}

The used concentration of MF for in vivo and in vitro studies was on the basis of previous studies. ${ }^{13}$

The mice were gavaged orally with DMSO (control) or MF $(100 \mathrm{mg} / \mathrm{kg})$ at 1 and $7 \mathrm{~h}$ prior to LPS instillation, which was 10 times as much as that for humans. To assess the potential in vivo toxicity of MF $(100 \mathrm{mg} / \mathrm{kg} /$ day $)$, pathological data on heart, liver and kidney was collected. MF $(100 \mathrm{mg} / \mathrm{kg} /$ day) could not induce heart, liver or kidney injury.

The J774A.1 cells were pretreated with PBS or MF $\left(10^{-5} \mathrm{M}\right)$ for $30 \mathrm{~min}$, and then the cells were treated with PBS or LPS $(100 \mathrm{ng} / \mathrm{mL})+$ ATP $(2 \mathrm{mM})$ for $6 \mathrm{~h}$.

\section{Haematoxylin \& Eosin (H\&E) Staining}

The paraffin-embedded lung, liver, heart and kidney tissue sections $(3 \mu \mathrm{m})$ were dewaxed using xylene, and stained using hematoxylin. Then, these sections were exposed to the gradient acid alcohol solution (hydrochloric acid and alcohol). Subsequently, the sections were dehydrated and cleared using anhydrous ethanol as well as xylene. Finally, these sections were observed under a light microscope and scored according to the previous description. ${ }^{14}$ 


\section{Lung Wet/Dry Ratio Determination}

After LPS stimulation for 12 hours, mice were euthanized and sacrificed by cervical dislocation. Then, the right lung was removed and the blood onto the surface was wiped away. Subsequently, the wet weight of right lung was weighed immediately. The specimens were incubated at $60^{\circ} \mathrm{C}$ for 96 hours. The dried tissues were weighed again and lung wet/dry ratio was recorded.

\section{MPO Activity Determination}

Lung tissues were homogenized and MPO activity was detected using a commercially available kit (ab105136, Abcam, Cambridge, MA, USA). Absorbance at $412 \mathrm{~nm}$ was determined.

\section{Immunohistochemical Staining}

The lung tissue sections were deparaffinized into water and dehydrated via gradient alcohol. After washing it with distilled water for $5 \mathrm{~min}, 3 \% \mathrm{H}_{2} \mathrm{O}_{2}$ was used to incubate the sections for $20 \mathrm{~min}$ to inactivate endogenous peroxidase. Following that, goat serum (10\%) was employed to block potential nonspecific binding sites of proteins for $30 \mathrm{~min}$ at room temperature. Then, the lung tissue sections were incubated with the primary antibody against NLRP3 at $4^{\circ} \mathrm{C}$ overnight and incubated with the secondary antibody at $37^{\circ} \mathrm{C}$ for $20 \mathrm{~min}$. Following that, these sections were added with HRP-labeled streptavidin protein working solution and developed using diaminobenzidine (DAB). These lung tissue sections were photographed under a light microscope.

\section{Cell Culture and Treatment}

The murine J774A.1 cells were obtained from the China Cell Line Bank (Beijing, China), which was cultured in DMEM medium with FBS $(10 \%)$ in a humidified atmosphere with $\mathrm{CO}_{2}(5 \%)$ at $37{ }^{\circ} \mathrm{C}$. J774A.1 cells were transfected with adenovirus harboring no overexpression sequence or Ad-NLRP3 $(\mathrm{MOI}=10)$ for $8 \mathrm{~h}$ to overexpress NLRP3. P65 siRNA was used to knock down the expression of P65 in J774A.1 cells. The J774A.1 cells were transfected using Lipofectamine 3000 (Thermo Fisher Scientific, Waltham, MA, USA) according to the manufacturer's instructions. Cells were challenged with LPS $(100 \mathrm{ng} / \mathrm{mL})$ and ATP $(2 \mathrm{mM})$ for $6 \mathrm{~h}$ after they reached $75 \%$ confluence. Experiments were carried out at least 3 times in duplicate.

\section{Real-Time PCR and Western Blot}

The lung tissues and iced macrophages were lysed using a RIPA buffer. The protein concentrations were detected by the BCA Protein Assay Kit. Following that, the protein extracts were loaded into sodium dodecyl sulfatepolyacrylamide gel electrophoresis (SDS-PAGE) gels and then transferred to a polyvinylidene fluoride (PVDF) membrane (Millipore, IPVH00010). Next, the membranes were incubated successively with primary antibodies and secondary antibodies. Finally, the membranes were reacted with ECL and the gray value was analyzed using Image $J$ gel analysis software. In this study, the cytosolic protein levels were normalized to $\beta$-actin while nuclear proteins were normalized to PCNA. As for real-time PCR, cDNA was synthesized from the total RNA extracted from the frozen lung tissues or macrophages by the Transcriptor First Strand cDNA Synthesis Kit. Quantitative RT-PCR analysis was then implemented using the LightCycler 480 SYBR Green 1 Master Mix. The mRNA levels in this study were calculated by the $2^{-\Delta \Delta \mathrm{Ct}}$ method, which were normalized to Gapdh. The primer sequences used are displayed in Table 1.

\section{Determination of IL-I $\beta$ and IL- I 8}

The homogenate of lung tissues and cell supernatant were centrifuged and collected. According to the instructions of the ELISA kits provided by Abcam, the levels of IL-1 $\beta$ (ab197742) as well as IL-18 (ab216165) in lung tissues and cell supernatant were measured.

\section{Statistical Analysis}

All data were presented as are expressed as mean \pm standard error of the mean (SEM) and analyzed using SPSS

Table I The Primers Used in Real-Time PCR

\begin{tabular}{|l|l|l|l|}
\hline Species & Gene & Forward Primer & Reverse Primer \\
\hline Mice & Tnf- $\alpha$ & ACTGAACTTCGGGGTGATCGGT & TGGTTTGCTACGACGTGGGCTA \\
Mice & II-6 & CCCCAATTTCCAATGCTCTCC & CGCACTAGGTTTGCCGAGTA \\
Mice & II- $\beta$ & AATGAAGGAACGGAGGAGCC & CTCCAGCCAAGCTTCCTTGT \\
Mice & Nlrp3 & CCAGAAACTGTGACTGTACATTGG & TTGGTCCCACACAAGCCTTT \\
Mice & Gapdh & ACTCCACTCACGGCAAATTC & TCTCCTATGGTGGTGACGACA \\
\hline
\end{tabular}


21.0 software. Comparisons between 2 groups were performed using an unpaired Student's $t$-test, while multiple comparisons among 3 or more groups were analyzed by one-way ANOVA, followed by post hoc Tukey's test. $P<$ 0.05 was regarded as statistical significance.

\section{Results}

\section{MF Protects Against LPS-Induced}

\section{Pathological Injury of Murine Lung Tissue}

To assess the effect of MF treatment on LPS-induced lung injury, wild-type mice were instilled intratracheally with LPS to mimic sepsis-induced lung injury. After 12 hours challenge, pathological injury was analyzed by H\&E staining. As shown in Figure 1A, LPS-induced pathological injury of murine lung tissue was significantly alleviated after MF pretreatment. Additionally, MF pretreatment also reduced LPS-induced lung edema, which was evidenced by the lower lung wet/ dry ratio (Figure 1B). Myeloperoxidase (MPO) is a lysosomal protein highly expressed in neutrophils, the activity of which represents the level of neutrophil infiltration in lung tissue. Our result disclosed that MPO activity in LPS+MF group was significantly lower than that in LPS group, indicating that MF was able to block neutrophil infiltration in lung tissue. These results suggest that MF possesses the potential to prevent LPSinduced lung injury.

\section{MF Inhibited the mRNA Levels of Proinflammatory Cytokines in Lung Tissues Treated with LPS}

The excessive activation of inflammatory response is one of the most vital pathological features during sepsisinduced $\mathrm{ALI}^{2}{ }^{2}$ Hence, we further detected the mRNA levels of proinflammatory cytokines including IL-1 $\beta$, IL6 and TNF- $\alpha$. As shown in Figure 2A-C, LPS instillation significantly promoted the mRNA expression of IL- $1 \beta$, IL6 and TNF- $\alpha$. However, the mRNA levels of these 3 proinflammatory cytokines were significantly decreased after MF pretreatment, indicating the anti-inflammatory action of MF.

\section{MF Suppressed the NLRP3 Inflammasome Activation in Lung Tissues Treated with LPS}

NLRP3 inflammasome involves NLRP3, Caspase-1 and ASC, the activation of which triggers the maturation and secretion of pro-inflammatory mediators including IL-1 $\beta$ and IL-18. ${ }^{15}$ Previous studies have proved that NLRP3 inflammasome activation is one of the main mechanisms contributing to ALI. Thus, we next detected the proteins and products associated with NLRP3 inflammasome. As displayed in Figure 3A, LPS challenge significantly promoted the protein expression of NLRP3 and GSDMD in
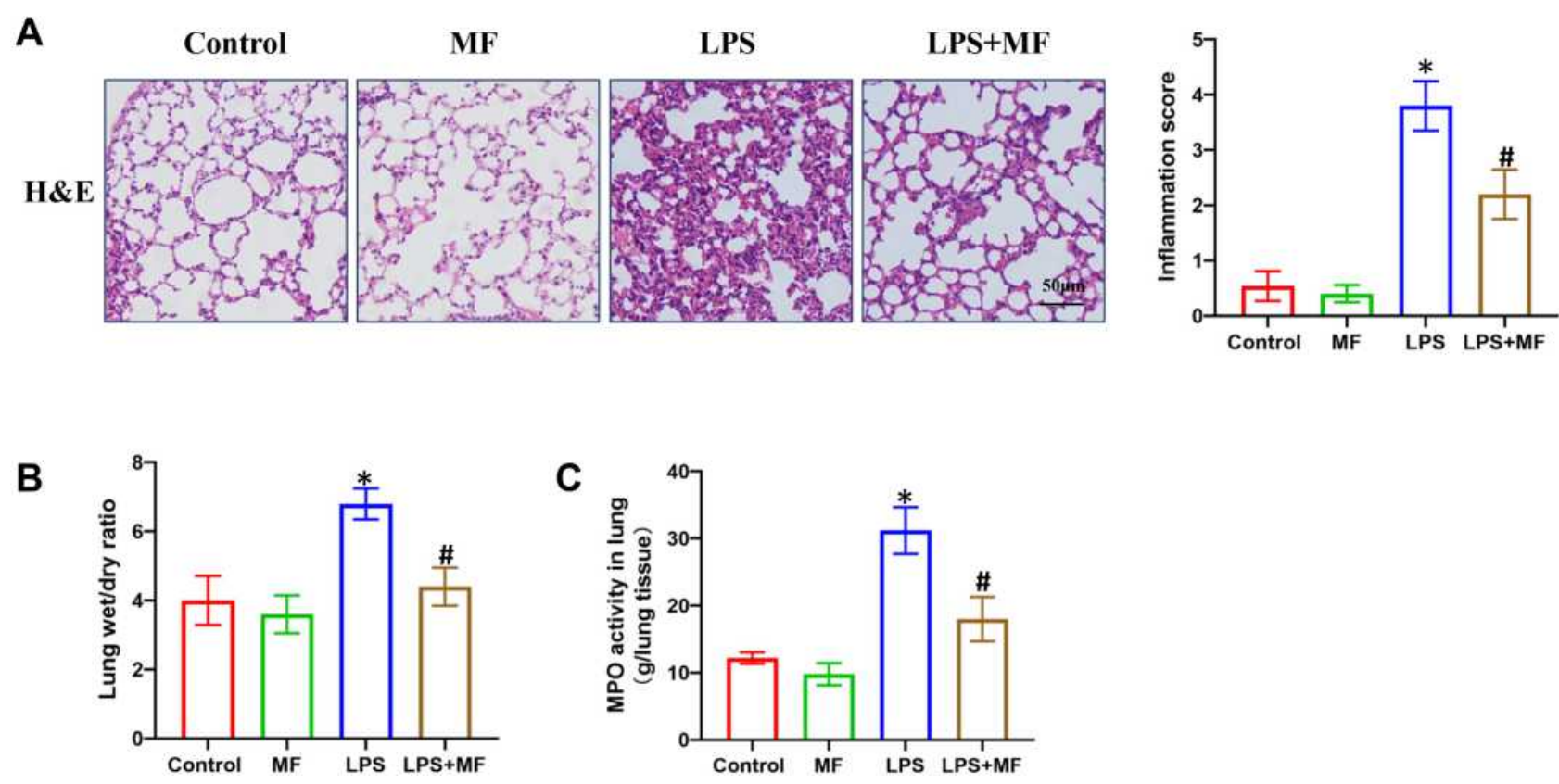

Figure I MF protects against LPS-induced pathological injury of murine lung tissue. (A) H\&E staining of murine lung tissues. (B) Lung wet/dry ratio in the indicated groups. (C) MPO activity in the indicated groups. ( $=5,{ }^{*} \mathrm{P}<0.05$ vs Control group, ${ }^{\#} \mathrm{P}<0.05$ vs LPS group). 

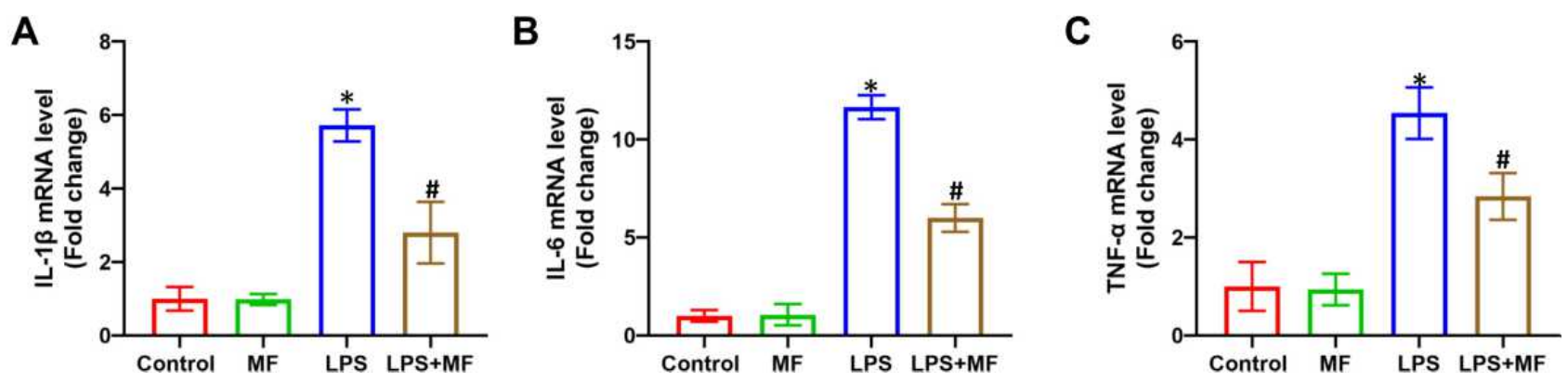

Figure 2 MF inhibited the mRNA levels of proinflammatory cytokines in lung tissues treated with LPS. (A-C) The mRNA levels of IL-I $\beta$, IL-6 and TNF- $\alpha$ in the indicated groups. ( $\mathrm{n}=5,{ }^{*} \mathrm{P}<0.05$ vs Control group, ${ }^{*} \mathrm{P}<0.05$ vs LPS group).

A
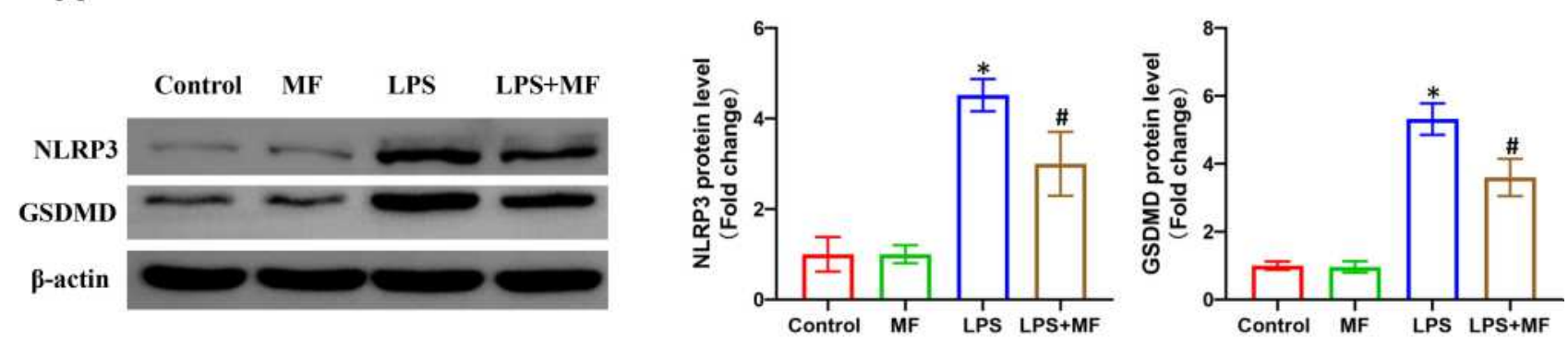

B

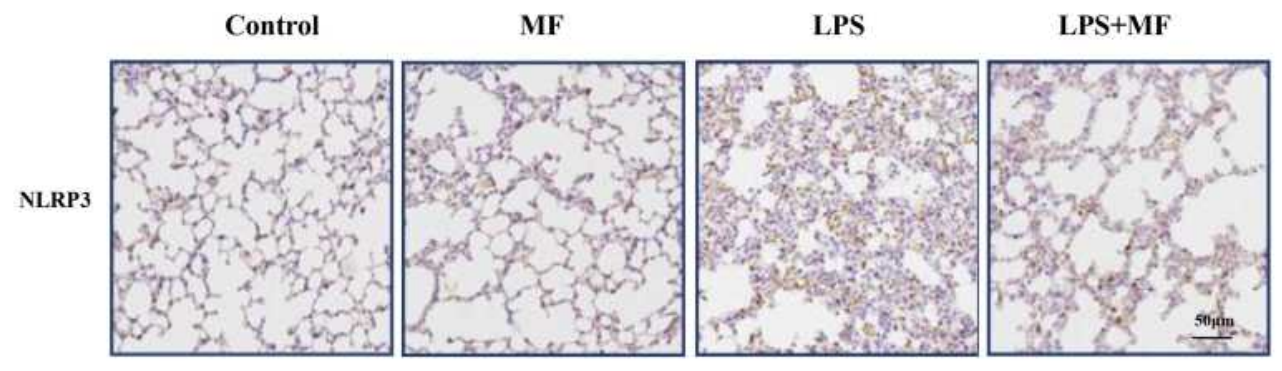

C
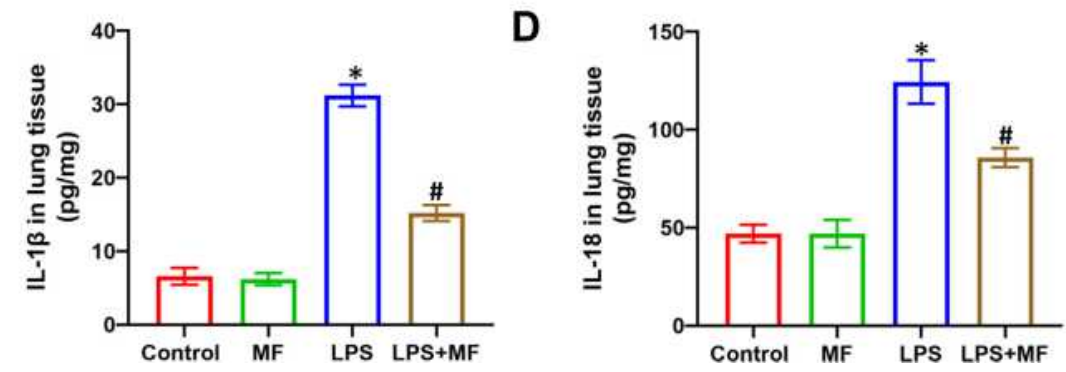

Figure 3 MF suppressed the NLRP3 inflammasome activation in lung tissues treated with LPS. (A) Western blots and statistical results for NLRP3 and GSDMD in lung tissues. (B) Immunohistochemistry staining for NLPR3 protein in the indicated groups. (C and $\mathbf{D})$ The levels of IL-I $\beta$ and IL-I8 in the lung tissues determined by ELISA. ( $\mathrm{n}=$ 5 , ${ }^{*} \mathrm{P}<0.05$ vs Control group, ${ }^{*} \mathrm{P}<0.05$ vs LPS group).

lung tissues, which could be reversed after MF pretreatment. The result from immunohistochemical staining further supported the inhibiting effect of MF on NLRP3
(Figure 3B). NLRP3 inflammasome activation could promote the cleavage and maturation of pro-IL-1 $\beta$ and pro-IL -18 . Therefore, we next measured the activated IL-1 $\beta$ and 
IL-18 in lung tissues using ELISA assay kit. The results (Figure 3C and D) showed MF pretreatment significantly decreased the levels of IL-1 $\beta$ and IL-18 in tissues, which further suggested the fact that MF could suppress the NLRP3 inflammasome activation in murine lung tissues.

\section{Macrophage-Specific NLRP3 Deficiency Counteracted the Protective Effects of MF in vivo}

To further figure out whether MF exerted pulmonary protection in an NLRP3-dependent manner, the macrophagespecific NLRP3-deficient mice were employed in the next experiments. As shown in Figure 4A-C, macrophagespecific NLRP3 deficiency also alleviated LPS-induced lung injury. However, MF pretreatment showed no antiinflammatory effects in NLRP3(-/-) mice challenged with LPS. Western blot (Figure 4D) showed that MF pretreatment further decreased the protein expression of GSDMD in NLRP3(-/-) mice challenged with LPS, hinting that MF may inhibit the level of GSDMD partially in a NLRP3dependent manner. Additionally, our results showed that MF pretreatment had no effects on the levels of IL-1 $\beta$ and IL-18 in NLRP3(-/-) mice challenged with LPS (Figure 4E). Intriguingly, no obvious lung edema between control and LPS groups in KO mice was observed. We speculated that there may be other signals contributing to the inflammatory response independent of NLRP3 (Figure 4B). Taken together, these results showed that MF may exert protective effects in a NLRP3-dependent manner.

\section{NLRP3 Overexpression Offset the Protective Effects of MF in vitro}

To further prove our hypothesis, we next employed adenovirus to overexpress NLRP3 in murine J774A.1 cells and observed the effects of MF on LPS-treated macrophages. Adenovirus infection significantly upregulated the expression of NLRP3 in macrophages (Supplement Figure 1A). As expected, MF pretreatment obviously inhibited the levels of IL-1 $\beta$ and IL-18 in cell supernatant (Figure 5A and $\mathrm{B}$ ) as well as the mRNA level of TNF- $\alpha$ (Figure 5C), and enhanced the cell viability (Figure 5D) of macrophages challenged with LPS for $6 \mathrm{~h}$, which was suppressed when NLRP3 was overexpressed. Additionally, although LPS treatment obviously increased the levels of ASC and caspase 1, NLRP3 overexpression or MF treatment had no effects on the levels of ASC and caspase 1, suggesting that the number of inflammasome may be saturated in the context of LPS stimulation at this concentration. MF downregulated NLRP3 to some extent, but the level of NLPR3 in LPS $+\mathrm{MF}+$ Ad-NLRP3 group was still higher than that in LPS group (Supplement Figure 1B). We assume that the number of inflammasome in LPS+Ad-NLRP3 and LPS+MF +Ad-NLRP3 group was determined by ASC but not NLRP3. Collectively, these data proved the fact that the protective effects of MF depended on NLRP3 at the cellular level.

\section{The Nuclear Translocation of the P65 and P50 Was Essential for the Protective Effects of MF}

Considering that LPS is one of the potent inducers of $\mathrm{NF}-\mathrm{\kappa B}$ signaling pathway and $\mathrm{NF}-\mathrm{\kappa B}$ also serves as a crucial transcription factor of NLRP3, we next explored whether MF could inhibit NF- $\mathrm{KB}$ signaling pathway. To begin with, the macrophages were transfected with $\mathrm{P} 65$ siRNA to inhibit the expression of P65. We found that P65 silence could significantly inhibit the mRNA level of NLRP 3 and decreased the levels of IL- $1 \beta$ and IL-18 in cell supernatant, indicating P65 is essential for NLRP3 inflammasome activation (Figure 6A-C). Next, we detected the protein levels of NF- $\mathrm{KB}$ subunits P65 and P50 in cytosolic extract and nuclear extract, respectively. The results showed that LPS could promote the nuclear translocation of P65 and P50, which could be reversed after MF pretreatment (Figure 6D), indicating that MF could inhibit NLRP3 inflammasome activation via blocking the nuclear translocation of the P65 and P50.

\section{Therapeutic Dose of MF in LPS-Induced ALI Displayed No Organic Toxicity}

To implement the clinical application of MF, we detected the effects of MF $(100 \mathrm{mg} / \mathrm{kg})$ on other organs including kidney, liver and heart. H\&E staining (Figure 7A) showed that MF $(100 \mathrm{mg} / \mathrm{kg})$ did not cause obvious tissue injury. Blood biochemical indexes showed that MF pretreatment had no effects on the levels of creatinine, ALT and CK$\mathrm{MB}$ in serum (Figure 7B-D), suggesting that MF (100 mg/ $\mathrm{kg}$ ) is the safe dose against LPS-induced ALI. 4-week MF intervention $(100 \mathrm{mg} / \mathrm{kg} / \mathrm{d})$ also displayed no organic toxicity, hinting that MF could be chosen as a dietary supplement (Supplement Figure 2A and B). 

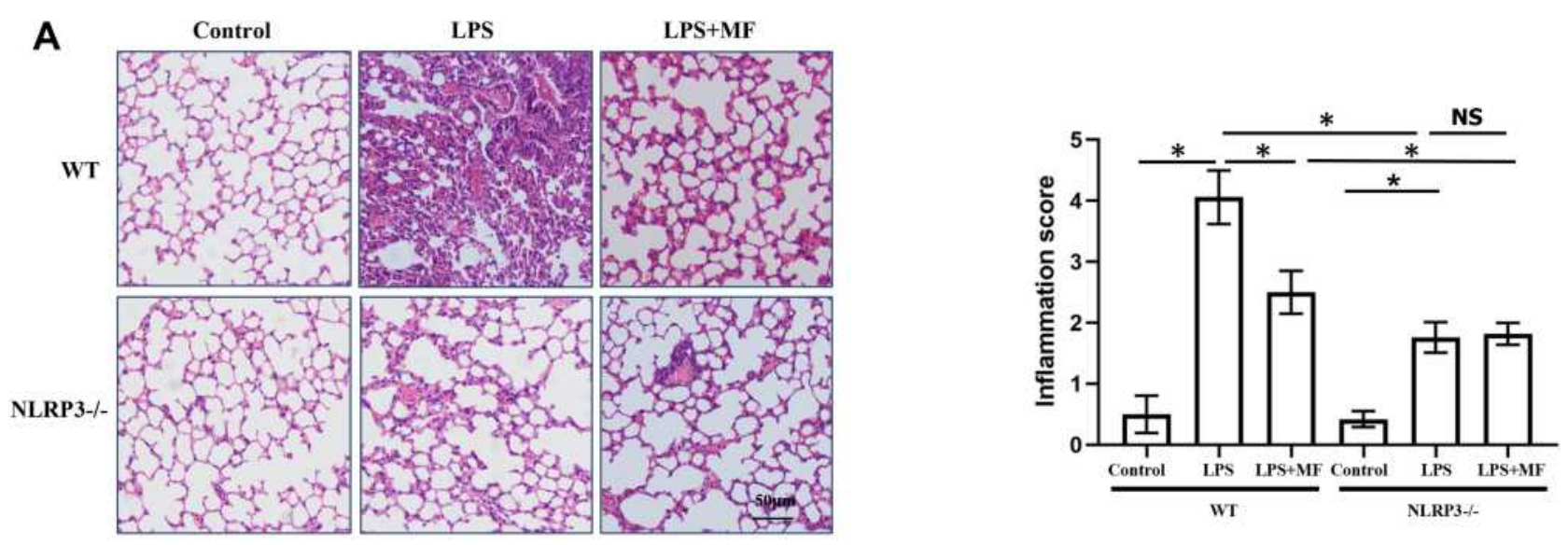

B

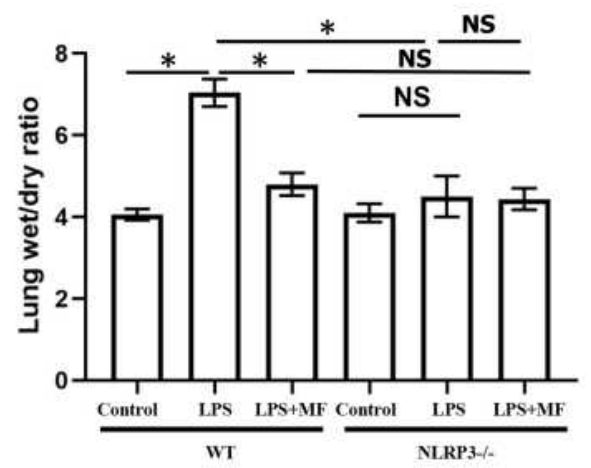

D

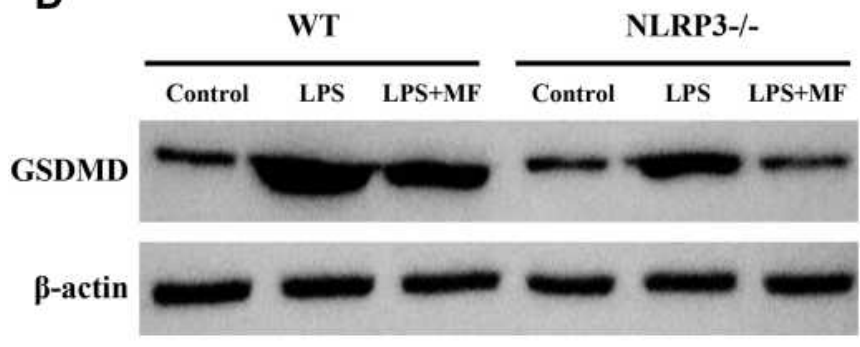

E

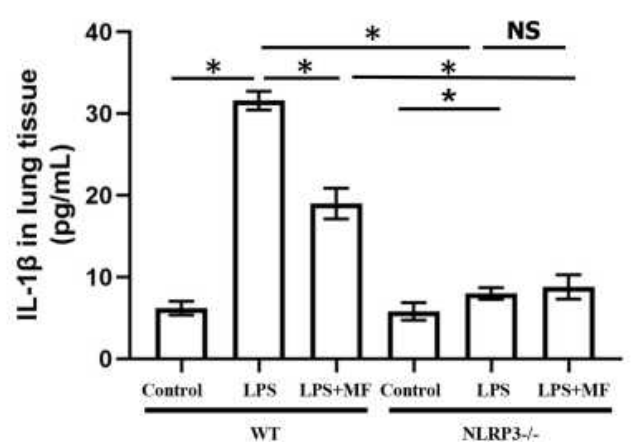

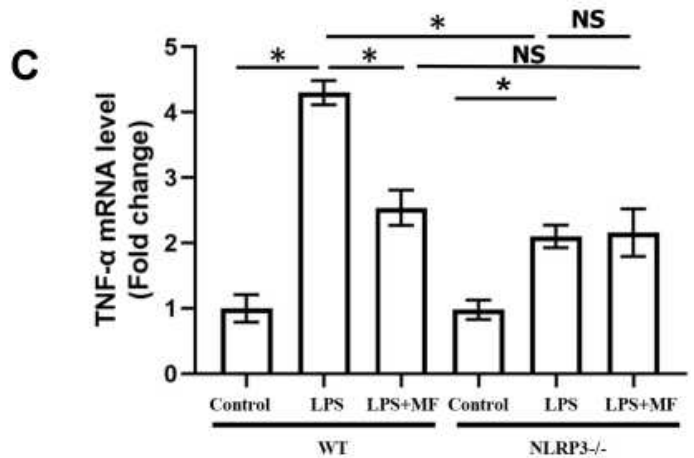

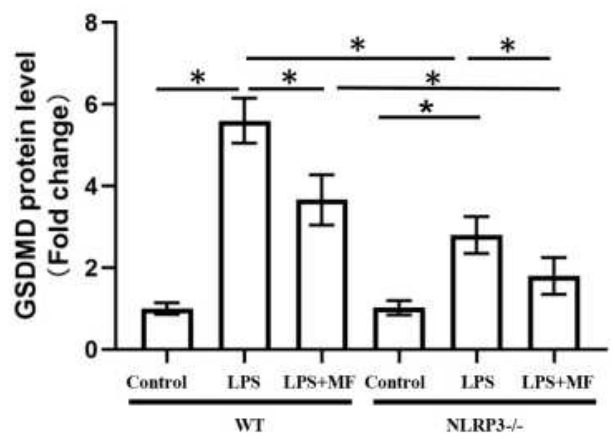

$\mathbf{F}$

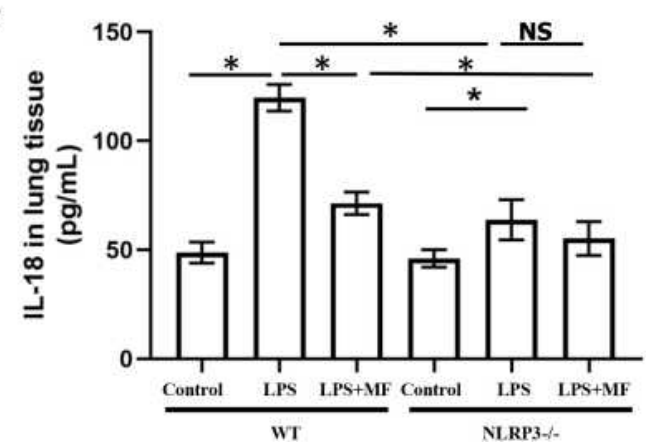

Figure 4 Macrophage-specific NLRP3 deficiency counteracted the protective effects of MF in vivo. (A) H\&E staining of murine lung tissues. (B) Lung wet/dry ratio in the indicated groups. (C) The mRNA level of TNF- $\alpha$ in the indicated groups. (D) Western blots and statistical results for GSDMD in lung tissues. (E and F) The levels of IL-I $\beta$ and IL- 18 in the lung tissues determined by ELISA. ( $n=5$, $* P<0.05$ vs indicated group).

Abbreviation: NS, no significance. 


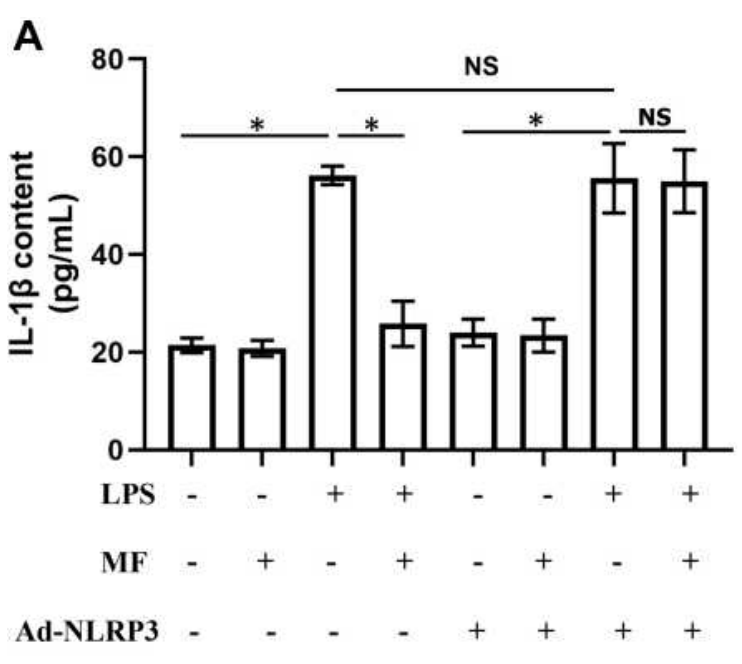

C

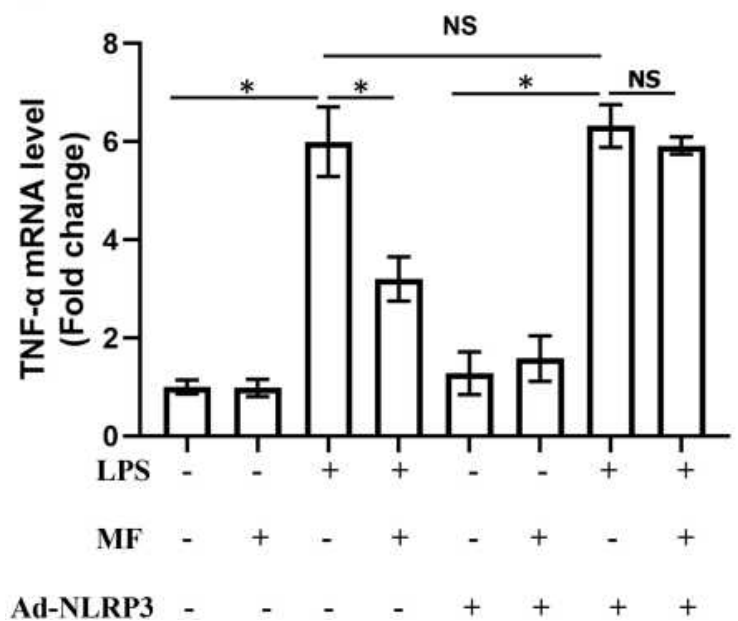

B

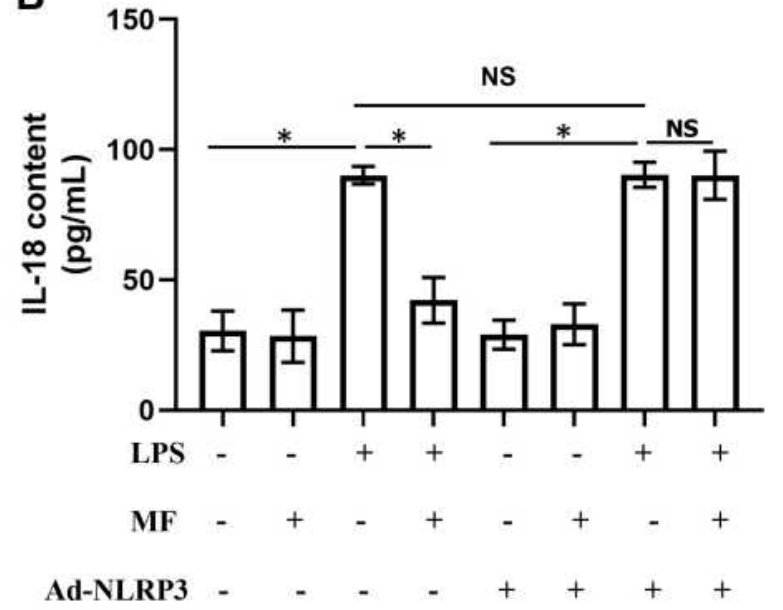

D

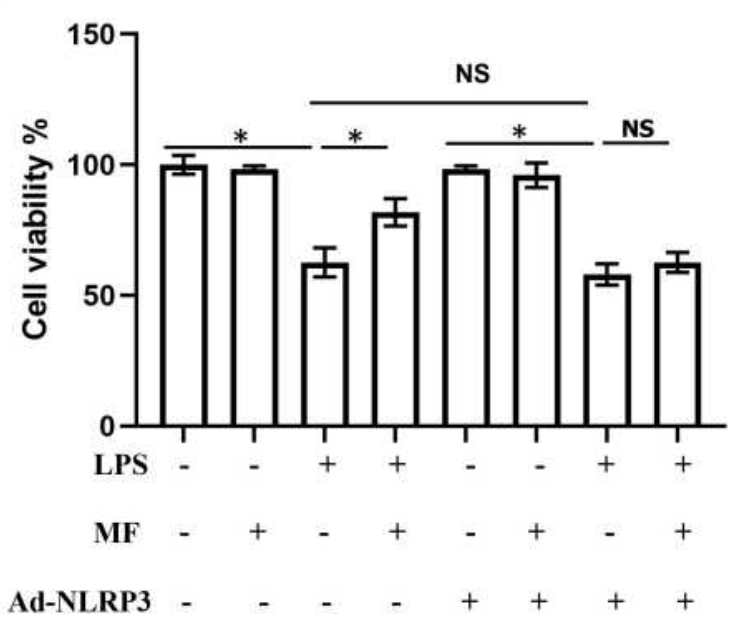

Figure 5 NLRP3 overexpression offset the protective effects of MF in J774A.I cells. (A and B) The levels of IL-I $\beta$ and IL-I8 in cell supernatant. (C) The mRNA level of TNF- $\alpha$ in the indicated groups. (D) Cell viability was measured by CCK 8 assay in the indicated group. ( $n=5$, $* \mathrm{P}<0.05$ vs indicated group).

Abbreviation: NS, no significance.

\section{Discussion}

The NLRP3 inflammasome activation of pulmonary alveolar macrophage aggravates the inflammatory response and promotes the development of ALI, giving rise to acute respiratory distress syndrome (ARDS) and even death. ${ }^{16}$ In the present study, we investigated the effects of MF on LPS-induced ALI based on in vivo and in vitro models, and found that MF pretreatment displays potential pulmonary protection. In terms of mechanism, MF pretreatment blocked the NLRP3 inflammasome activation and inhibited pyroptosis of macrophages in a P65-dependent manner (Figure 8).

MF is one of the main components derived from mango fruits, which has been extensively used because it displays hypotoxicity and owns a broad spectrum pharmacological properties including antiinflammatory anti-diabetic, anti-oxidative, anti-aging, anti-viral and anti-cancer effects. ${ }^{10}$ For example, in arsenic-induced lung injury, MF suppressed inflammatory cell infiltration and restored the antioxidant balance by upregulating Nrf2-HO1 signaling pathway. ${ }^{17}$ In human ovarian carcinoma OVCAR3 cells, MF significantly decreased viability of cancer cells, and increased the sensitivity of cancer cells to cisplatin. ${ }^{18}$ Similarly, MF also exerts critical and promising effects on respiratory disease. In detail, MF could inhibit the expression of transforming growth factor- $\beta 1$ (TGF- $\beta 1$ ) and $\alpha$-smooth muscle actin ( $\alpha$-SMA) in bleomycin- 
A

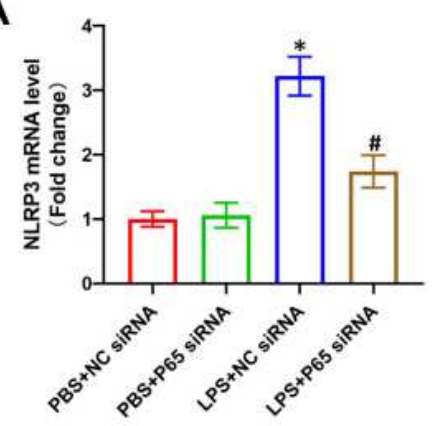

D
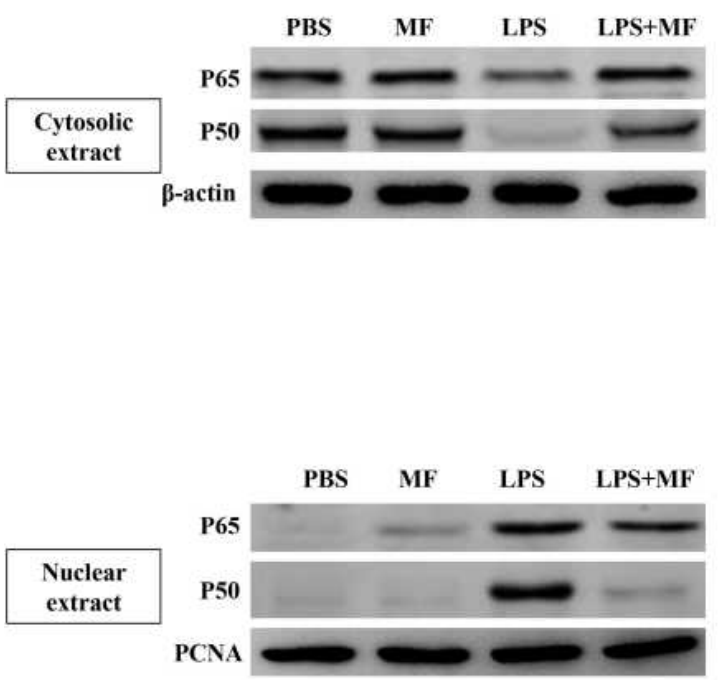

B
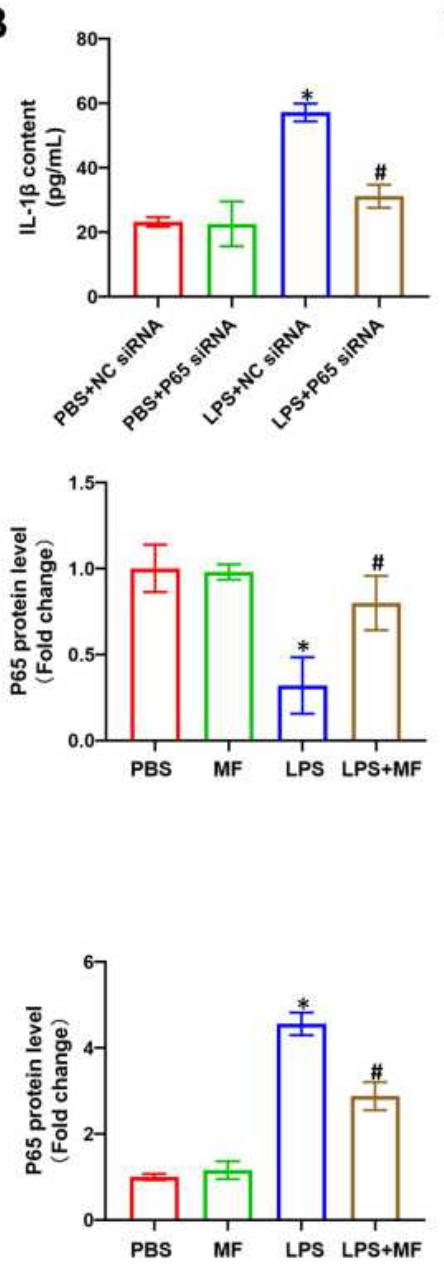

C
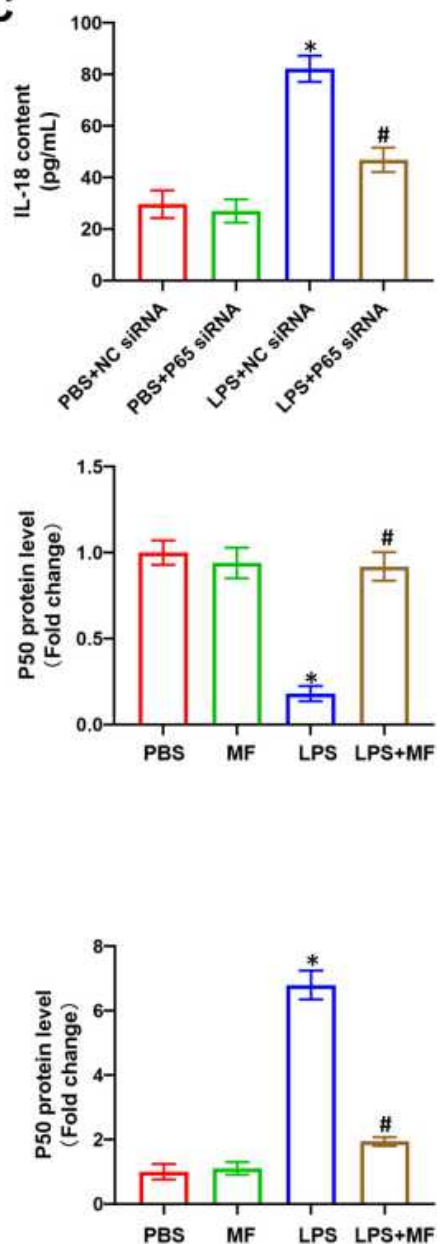

Figure 6 The nuclear translocation of the P65 and P50 was essential for the protective effects of MF in J774A.I cells. (A) The mRNA level of NLRP3 in the indicated groups. $(B$ and $\mathbf{C})$ The levels of IL-I $\beta$ and IL- 18 in cell supernatant, $\left(n=5,{ }^{* P}<0.05\right.$ vs PBS+NC siRNA group, ${ }^{\#} \mathrm{P}<0.05$ vs LPS $+\mathrm{NC}$ siRNA group). (D) Western blots and statistical results for P65 and P50 in cytosolic extract and nuclear extract, $\left(n=5, * P<0.05\right.$ vs Control group, ${ }^{*} P<0.05$ vs LPS group).

induced pulmonary fibrosis experimental model by inhibiting TLR4/P65-mediated inflammatory response and TGF- $\beta 1 / \mathrm{Smad} 2 / 3$-mediated epithelial-mesenchymal transition. ${ }^{19}$ In the current study, we discovered that MF pretreatment could obviously decrease pulmonary inflammatory response, fibrosis and edema in LPS-treated lung tissues and alveolar macrophages. Based on these data, we hypothesized that MF may serve as a possible candidate against ALI.

Pyroptosis is a type of programmed cell death (PCD) which is accompanied by cell rupture and release of inflammatory factors. ${ }^{20}$ Pyroptosis is usually triggered by inflammasomes and its downstream GSDMD, which must be cleaved via caspase-1 or caspase-11 pathway before if functions. ${ }^{21,22}$ Till now, a total of 5 types of inflammasomes have been identified, namely NLRP1 inflammasome, NLRP3 inflammasome, NLRC4 inflammasome, IPAF inflammasome and absent in melanoma 2 (AIM2) inflammasome. ${ }^{23}$ Generally, one inflammasome generally contains an apoptosis-associated speck-like protein containing CARD, ASC, caspase protease, and NLR family protein (such as NLRP1) or HIN200 family protein (such as AIM2). ${ }^{4,24,25}$ Kovarova et al $^{26}$ disclosed that assembly of the NLRP1 inflammasome could initiate pyroptosis, which then gives rise to a self-amplifying cascade of lung injury triggered by a toxin from anthrax, eventually causing catastrophic consequences. In LPS-induced ALI in old mice, inhibiting NLRC4 inflammasome activation by metformin via AMPK could alleviate lung injury and reduce the levels of pro-inflammatory factors in the bronchoalveolar lavage fluid. ${ }^{27}$ AIM2 inflammasome also plays a critical role in ALI. AIM2-deficient mice challenged 
A

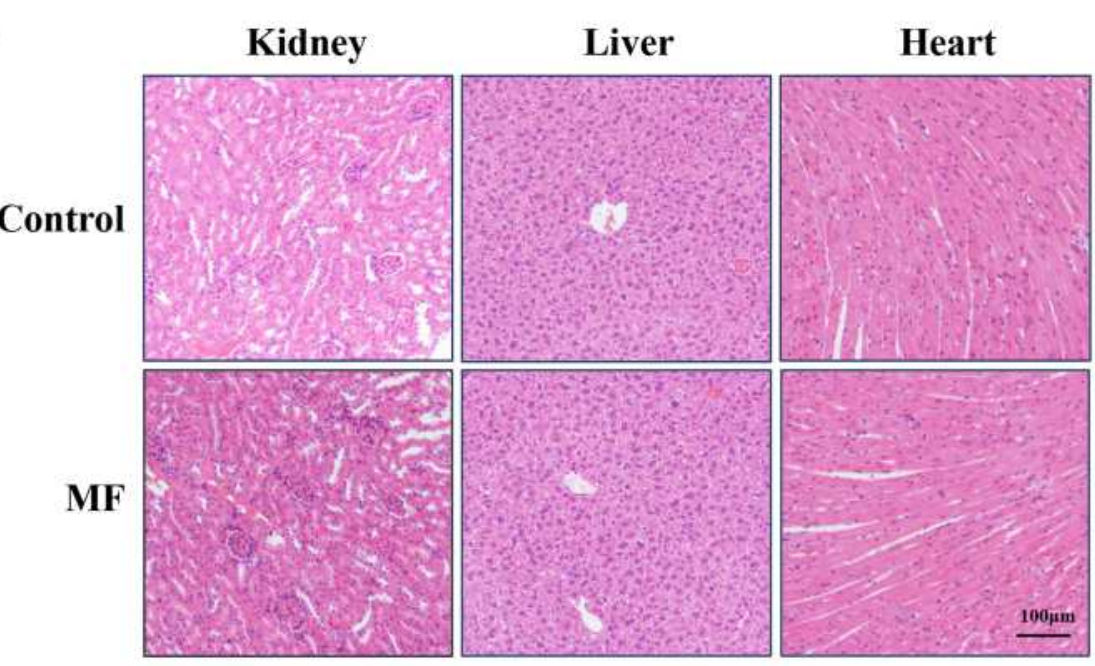

B

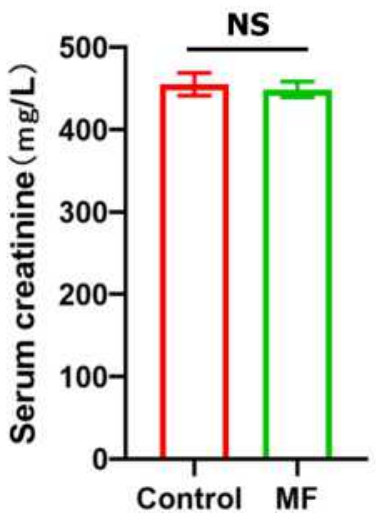

C

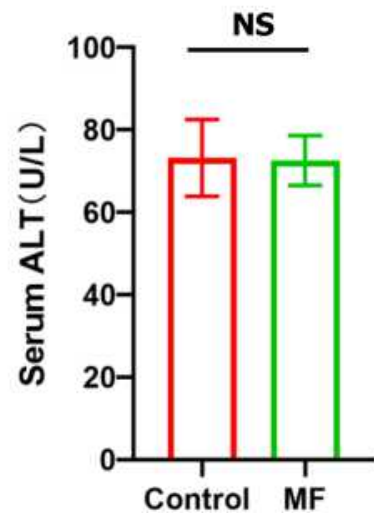

D

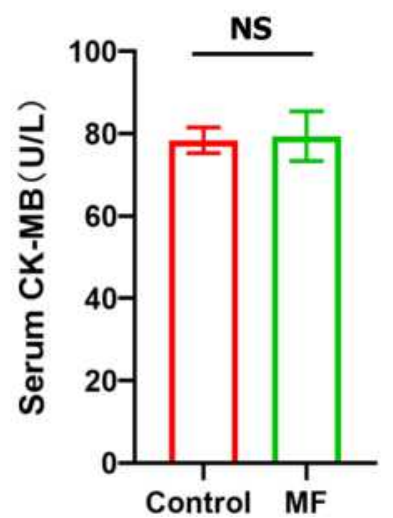

Figure 7 Therapeutic dose of MF displayed no organic toxicity. (A) H\&E staining of murine kidney, liver and heart tissues. (B-D) The levels of creatinine, ALT and CK-MB in serum. $(n=5)$.

Abbreviation: NS, no significance.

with influenza A virus displayed alleviative lung injury and improved survival compared with wild-type mice. $^{28}$ We recently reported that NLRP3 inflammasome-dependent macrophage pyroptosis may also contribute to lung injury caused by sepsis. ${ }^{6}$ Hence, inflammasome inhibition may serve as a pharmacological target for relieving ALI induced by infection or trauma. In this study, we reported that NLRP3 inflammasome and pyroptosis could be inhibited by MF in LPS-treated lung tissues and macrophages. Meanwhile, we found that MF could inactivate NLRP3 inflammasome in a P65-dependent manner in macrophages. Previous study showed that P65 may act as a transcription factor for NLRP3. In keeping with this, in the present study, we found that P65 silencing may decrease the mRNA level of NLRP3. Thus, we speculate that MF may reduce the expression of NLRP3 by inhibiting the nuclear translocation of P65.

In conclusion, our study showed that MF may attenuate LPS-induced ALI by inhibiting NLRP3 inflammasome activation in a P65-dependent manner. Therefore, the inhibition of P65-mediated NLRP3 inflammasome activation and pyroptosis in macrophages by MF may act as a possible therapeutic strategy against ALI and ARDS. However, more studies aiming to illustrate the preclinical value of MF in other animal models are of great importance. 


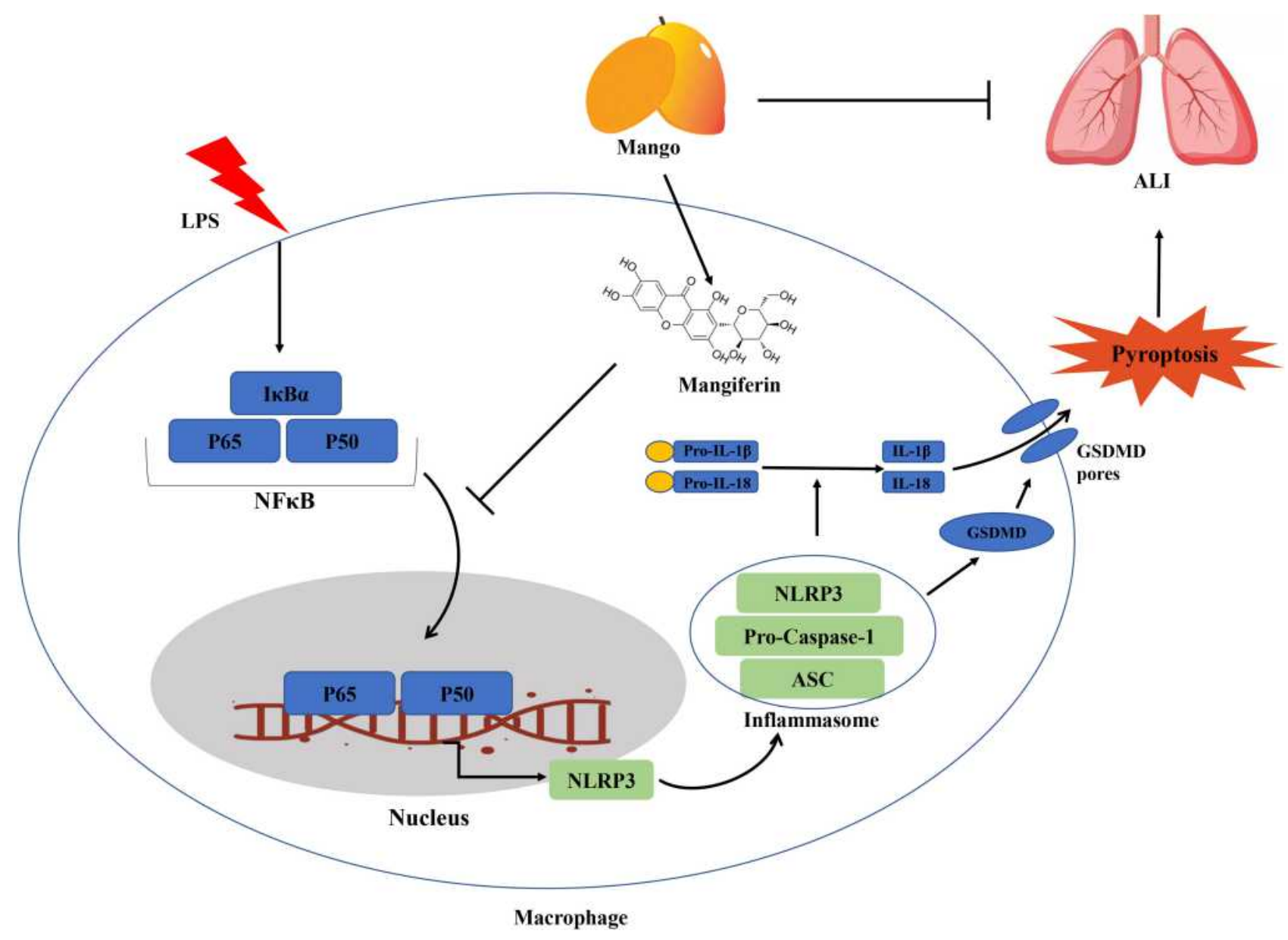

Figure 8 The possible mechanisms for the protective role of MF in LPS-induced ALI.

\section{Data Sharing Statement}

All data that support the findings in this study are available from the corresponding author upon reasonable request.

\section{Author Contributions}

Li Ning, Xiong Rui, Wang Bo, Geng Qing contributed to conception, designed experiments and were responsible for the whole work; Xiong Rui, He Ruyuan, Liu Bohao performed experiments; Li Ning and Xiong Rui analyzed experimental results and wrote the manuscript. All authors contributed to data analysis, drafting or revising the article, have agreed on the journal to which the article will be submitted, gave final approval for the version to be published, and agree to be accountable for all aspects of the work.

\section{Funding}

This work was supported by grants from the National Natural Science Foundation of China (Nos. 81800343,
81770095) and the Fundamental Research Funds for the Central Universities (No. 2042021kf0081).

\section{Disclosure}

The authors declare no conflicts of interest.

\section{References}

1. Matthay M, Arabi Y, Siegel E, et al. Phenotypes and personalized medicine in the acute respiratory distress syndrome. Intensive Care Med. 2020;46(12):2136-2152. doi:10.1007/s00134-020-06296-9

2. Matthay M, Ware L, Zimmerman G. The acute respiratory distress syndrome. J Clin Invest. 2012;122(8):2731-2740. doi:10.1172/ JCI60331

3. Zhang D, Song D, Shi L, et al. Mechanisms of interactions between lung-origin telocytes and mesenchymal stem cells to treat experimental acute lung injury. Clin Transl Med. 2020;10(8):e231. doi:10.1002/ $\operatorname{ctm} 2.231$

4. Feng Y, Tan Z, Wu L, Dong F, Zhang F. The involvement of NLRP3 inflammasome in the treatment of Alzheimer's disease. Ageing Res Rev. 2020;64:101192. doi:10.1016/j.arr.2020.101192

5. Chauhan D, Vande Walle L, Lamkanfi M. Therapeutic modulation of inflammasome pathways. Immunol Rev. 2020;297(1):123-138. doi:10.1111/imr.12908 
6. Ning L, Wei W, Wenyang J, Rui X, Qing G. Cytosolic DNA-STINGNLRP3 axis is involved in murine acute lung injury induced by lipopolysaccharide. Clin Transl Med. 2020;10(7):e228. doi:10.1002/ ctm2.228

7. Ying Y, Mao Y, Yao M. NLRP3 inflammasome activation by MicroRNA-495 promoter methylation may contribute to the progression of acute lung injury. Mol Ther Nucleic Acids. 2019;18:801-814. doi:10.1016/j.omtn.2019.08.028

8. Imran M, Arshad M, Butt M, Kwon J, Arshad M, Sultan M. Mangiferin: a natural miracle bioactive compound against lifestyle related disorders. Lipids Health Dis. 2017;16(1):84. doi:10.1186/ s12944-017-0449-y

9. Zhang L, Huang C, Fan S. Mangiferin and organ fibrosis: a mini review. Biofactors. 2020.

10. Naraki K, Rezaee R, Mashayekhi-Sardoo H, Hayes A, Karimi G. Mangiferin offers protection against deleterious effects of pharmaceuticals, heavy metals, and environmental chemicals. Phytother Res. 2020;35(2):810-822. doi:10.1002/ptr.6864

11. Fan K, Ma J, Xiao W, et al. Mangiferin attenuates blast-induced traumatic brain injury via inhibiting NLRP3 inflammasome. Chem Biol Interact. 2017;271:15-23. doi:10.1016/j.cbi.2017.04.021

12. Song J, Li J, Hou F, Wang X, Liu B. Mangiferin inhibits endoplasmic reticulum stress-associated thioredoxin-interacting protein/NLRP3 inflammasome activation with regulation of AMPK in endothelial cells. Metabolism. 2015;64(3):428-437. doi:10.1016/j. metabol.2014.11.008

13. Dong M, Li L, Li G, et al. viaMangiferin protects against alcoholic liver injury suppression of inflammation-induced adipose hyperlipolysis. Food Funct. 2020;11(10):8837-8851. doi:10.1039/ D0FO01436B

14. Duan J, Zhou Y, Zhou A, et al. Calcitonin gene-related peptide exerts anti-inflammatory property through regulating murine macrophages polarization in vitro. Mol Immunol. 2017;91:105-113. doi:10.1016/j. molimm.2017.08.020

15. Surabhi S, Cuypers F, Hammerschmidt S, Siemens N. The role of NLRP3 inflammasome in pneumococcal infections. Front Immunol. 2020;11:614801. doi:10.3389/fimmu.2020.614801

16. Zhang Y, Li X, Grailer J, et al. Melatonin alleviates acute lung injury through inhibiting the NLRP3 inflammasome. J Pineal Res. 2016;60 (4):405-414. doi:10.1111/jpi.12322
17. Mahalanobish S, Saha S, Dutta S, Sil P. Mangiferin alleviates arsenic induced oxidative lung injury via upregulation of the Nrf2-HO1 axis. Food Chem Toxicol. 2019;126:41-55. doi:10.1016/j.fct.2019.02.022

18. Zou B, Wang H, Liu Y, et al. Mangiferin induces apoptosis in human ovarian adenocarcinoma OVCAR3 cells via the regulation of Notch3. Oncol Rep. 2017;38(3):1431-1441. doi:10.3892/or.2017.5814

19. Jia L, Sun $\mathrm{P}, \mathrm{Gao} H$, et al. Mangiferin attenuates bleomycin-induced pulmonary fibrosis in mice through inhibiting TLR4/p65 and TGF31/Smad2/3 pathway. J Pharm Pharmacol. 2019;71(6):1017-1028. doi:10.1111/jphp.13077

20. Wu J, Sun J, Meng X. Pyroptosis by caspase-11 inflammasome-gadermin D pathway in autoimmune diseases. Pharmacol Res. 2021;165:105408. doi:10.1016/j.phrs.2020.105408

21. Zhang Z, Zhang Y, Lieberman J. Lighting a fire: can we harness pyroptosis to ignite antitumor immunity? Cancer Immunol Res. 2021;9(1):2-7. doi:10.1158/2326-6066.CIR-20-0525

22. Kist M, Vucic D. Cell death pathways: intricate connections and disease implications. EMBO J. 2021;40(5):e106700. doi:10.15252/ embj. 2020106700

23. Camilli G, Blagojevic M, Naglik J, Richardson J. Programmed cell death: central player in fungal infections. Trends Cell Biol. 2020;31 (3):179-196. doi:10.1016/j.tcb.2020.11.005

24. Tang R, Xu J, Zhang B, et al. Ferroptosis, necroptosis, and pyroptosis in anticancer immunity. $J$ Hematol Oncol. 2020;13(1):110. doi:10.1186/s13045-020-00946-7

25. Abu Khweek A, Amer A. Pyroptotic and non-pyroptotic effector functions of caspase-11. Immunol Rev. 2020;297(1):39-52. doi:10.1111/imr. 12910

26. Kovarova M, Hesker P, Jania L, et al. NLRP1-dependent pyroptosis leads to acute lung injury and morbidity in mice. J Immunol. 2012;189(4):2006-2016. doi:10.4049/jimmunol.1201065

27. He Y, Xu K, Wang Y, et al. AMPK as a potential pharmacological target for alleviating LPS-induced acute lung injury partly via NLRC4 inflammasome pathway inhibition. Exp Gerontol. 2019;125:110661. doi:10.1016/j.exger.2019.110661

28. Zhang H, Luo J, Alcorn J, et al. AIM2 inflammasome is critical for influenza-induced lung injury and mortality. J Immunol. 2017;198 (11):4383-4393. doi:10.4049/jimmunol.1600714
Journal of Inflammation Research

\section{Publish your work in this journal}

The Journal of Inflammation Research is an international, peerreviewed open-access journal that welcomes laboratory and clinical findings on the molecular basis, cell biology and pharmacology of inflammation including original research, reviews, symposium reports, hypothesis formation and commentaries on: acute/chronic inflammation; mediators of inflammation; cellular processes; molecular mechanisms; pharmacology and novel anti-inflammatory drugs; clinical conditions involving inflammation. The manuscript management system is completely online and includes a very quick and fair peerreview system. Visit http://www.dovepress.com/testimonials.php to read real quotes from published authors. 